now have Brandt and Chapman's Introduction to Comets. Let me say straight away that a very good introduction it is too; a worthy and timely fourth addition to the list. The authors have conveyed the excitement of modern comet work, the pace of the data flow and the originality of many of the new theoretical approaches. They are excellent recruiting agents for the subject.

The book starts by placing comets in the historical perspective and reviews many of our modern ideas about comets by discussing how scientists first approached, for

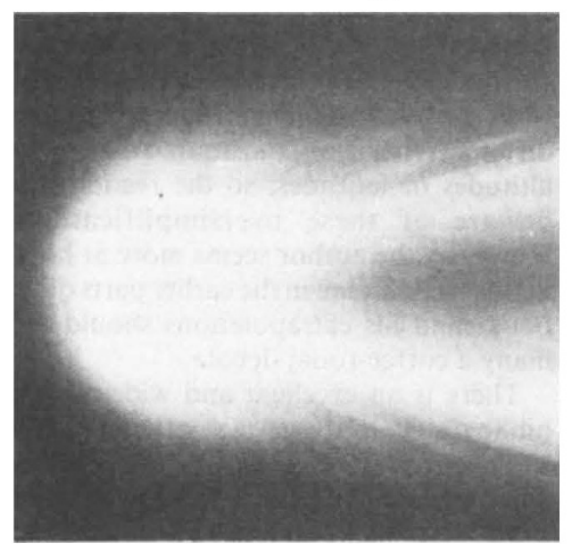

Halley's head, photographed on May 8, 1910, from the Hale Observatory.

example, cometary spectroscopy and the interactions of comets with the solar wind. The second section deals in depth with the dynamics and structure of comets and looks at the models that have been proposed for the chemical reactions and dust emission processes. The origins of comets and their decay into meteor streams are also considered. The third section reviews the enormous advances that have come from the investigation of some of the recent bright comets, such as Kohoutek, Kobayashi-Berger-Milon and West. It also discusses the possible spacecraft missions to comets. The last section briefly touches on comet germs and impacts, and the place of comets in literature and art.

The book is extremely well illustrated and delves into the nature of comets with sufficient detail to satisfy the average undergraduate and postgraduate reader. Those seeking more, are efficiently led to an impressive list of publications in the "suggested reading" section.

I think the authors have used their 246 pages most effectively. For my taste I would have put in more than three pages on origin mechanisms, and would have laid more emphasis on orbital evolution, decay process and size variation. Also the section on cometary missions seems to have been written long before the publication date, in the rosy times when NASA were still actively planning cometary missions. The people who are going to Halley in 1986 don't get a mention.

David $W$. Hughes is a Lecturer in Physics and Astronomy at the University of Sheffield.

\title{
Progress and prospects in solar physics
}

\section{J. C. Brown}

Solar Active Regions: A Monograph from Skylab Solar Workshop III. Edited by Frank Q. Orrall. Pp.350. ISBN 0-87081-085-5. (Colorado Associated University Press: 1981.) $\$ 17.50$.

IDEALLY the trilogy of Skylab Solar Workshop proceedings would have progressed from the tranquillity of Coronal Holes through Active Regions to achieve its nexus in Solar Flares, thereby exhibiting the physical progression of solar phenomena. In reality even the comparative opulence of workshop funds, albeit a tiny fraction of mission costs, did not permit this degree of coordination. Nevertheless the substantial overlap of participant lists means that the three workshop volumes together contain useful interdisciplinary seeds for the solar activity student to cultivate. Benefiting perhaps from previous experience, this third volume is the most readable.

The editor opens with a thorough overview of workshop results from each of the five teams who contribute about two detailed chapters each. A recurring theme throughout is theoretical and observational modelling of static loops of which the corona is now held mainly to consist. It appears to be generally accepted that spectral analysis can shed only dim light on details of loop heating mechanisms, though scaling laws permit comparison of the magnitude of heating in relation to loops of different lengths and pressures. Aside from these static analyses, the work includes a lengthy theoretical discussion of gas flows and thermal instability in loops, complemented by two chapters on observations of dynamic phenomena at chromospheric/coronal levels and near the photosphere, the latter invoking data from OSO-8 as well as from Skylab.

For the non-specialist the chapters of greatest interest will be those on theoretical coronal heating mechanisms and on spectral diagnostics, both of which are lucidly presented. The former contains much on energy release in plasmas which will interest plasma astrophysicists in general, while the latter concisely reviews the principles of spectrum line diagnostics for plasma temperatures, densities and differential emission measures. A frank assessment of progress made and of prospects for future work on active regions completes the monograph.

As usual this workshop raised more questions than it resolved, reiterating the stark solar message for cosmic astronomers of just how difficult astrophysical problems become when the object of study is close enough for detailed scrutiny. The Skylab Workshop series presented a unique opportunity for thrashing out both theoretical and observational progress in important specialized problems. It is to be hoped that the sorry current state of NASA funding does not preclude such in-depth attacks on data from more recent missions such as Solar Maximum.

J. C. Brown is Reader in Astronomy at the University of Glasgow.

\section{Sex differentiated}

\section{W. K. Whitten}

Mechanisms of Sex Differentiation in Animals and Man. Edited by C.R. Austin and R.G. Edwards. Pp.603. ISBN 0-12-068540-X. (Academic: 1981.) £37, $\$ 89$.

IN 1970, Harris and Edwards edited the Royal Society's discussion Sex Determination, and in 1972 a chapter by Short entitled Sex Determination and Differentiation appeared in Reproduction in Mammals, edited by Austin and Short, and published by Cambridge University Press. Now Austin and Edwards restrict their title to Sex Differentiation and argue that determination is the initial step in homotypic differentiation. But this is not accepted by some of their contributors, so the editors conclude that the argument is unresolved - a healthy attitude, emphasizing the need to standardize terminology.

This volume of 12 chapters written by specialists is intended as a text for advanced students and researchers. Lyon, Cattanach and Charlton give superb descriptions of testicular feminization, $\mathrm{XY}$ female wood lemmings, and sex-reversed and hypogonodal mice. The study of these conditions and the $\mathrm{H}-\mathrm{Y}$ antigen, described by Wachtel and Koo, have contributed greatly to the recent explosion in our understanding of sex differentiation. There are excellent chapters on embryonic development and function, postnatal growth of gonads and sex characters from the clinical point of view, and the many defects in determination and differentiation in human beings. The structure of the gonad, however, is followed only as far as initiation of meiosis. I assume that this is because folliculogenesis and gametogenesis are covered in other recent books such as Peters and McNatty's The Ovary published by the University of California Press in 1980. There is a review of $\mathrm{X}$-chromosome inactivation, but unfortunately no contribution by Ohno who anticipated many of the recent advances.

Benirschke adequately reviews freemartins and hermaphrodites of large animals, but his treatment of mosaics and chimaeras of mice is poor. Apparently he 